\title{
State-of-the-art basic and clinical science of Kawasaki disease
}

The 9th International Kawasaki Disease Symposium

10-12 April 2008, Taipei, Taiwan

\author{
Mei-Hwan Wu' ${ }^{1}$, Yosikazu Nakamura2 ${ }^{2}$ Jane C Burns 3 , Anne H Rowley4, \\ Kei Takahashi ${ }^{5}$, Jane W Newburger ${ }^{6}$, Masahiro Ishii ${ }^{7} \&$ Brian W McCrindle ${ }^{8 \dagger}$ \\ ${ }^{\dagger}$ Author for correspondence \\ ${ }^{1}$ National Taiwan University Hospital, Department of Pediatrics, Taipei, Taiwan \\ 2Jichi Medical University, Department of Public Health, Shimotsuke, Japan \\ 3University of California San Diego, Department of Pediatrics, Rady Children's \\ Hospital, San Diego, CA, USA \\ ${ }^{4}$ Northwestern University Feinberg School of Medicine, Department of Pediatrics \& \\ Microbiology/lmmunology, The Children's Memorial Hospital, Chicago, IL, USA \\ ${ }^{5}$ Toho University Medical Center Ohashi Hospital, Department of Pathology, \\ Tokyo, Japan \\ 6Harvard University, Department of Pediatrics, Department of Cardiology, \\ Children's Hospital of Boston, Boston, MA, USA \\ ${ }^{7}$ Kitasato University, Department of Pediatrics, Sagamihara, Japan \\ 8The Hospital for Sick Children, 555 University Avenue, Toronto, Ontario, \\ M5G 1X8, Canada \\ Tel.: +1 416813 7610; Fax: +1 416813 7547; E-mail: brian.mccrindle@sickkids.ca
}

Kawasaki disease (KD) has an increasing incidence worldwide. Taiwan has been estimated to have the third highest incidence of $K D$, with annual rates of approximately 70 per 100,000 children aged below 5 years. Therefore, after the first eight International KD Symposia (IKDS), which were alternately held in Japan or the USA, the 9th IKDS marked the first time that this event has been held at the Grand Hotel, Taipei, Taiwan, 28 years after the diagnosis of the first patient in Taiwan. This 9th IKDS attracted more than 400 participants from 22 countries and 244 abstracts were presented from all continents. The meeting was chaired by $\mathrm{Hung}-\mathrm{Chi}$ Lue (D epartment of Pediatrics, $N$ ational Taiwan University H ospital, Taipei, Taiwan), with vice-chair and secretary general Mei-H wan Wu (D epartment of Pedia trics, $\mathrm{N}$ ational Tai wan U niversity $\mathrm{H}$ ospital). The abstracts and summaries of invited lectures are now avail lable at [101].

Eighteen invited lectures were given that highlighted the latest research on $K D$. The symposium began with the
$\mathrm{H}$ ideko O gawa memorial lecture and state-of-the-art lectures, which covered topics including the surgical efficacy of using the internal thoracic artery for coronary artery bypass, the latest genomic data on KD susceptibility and epidemiological updates illustrating the general trend of increasing incidence. $\mathrm{KD}$ is now a well-recognized condition in some Asian countries where it had previously been widely overlooked, such as India and Mongolia. Pathological findings of KD were then detailed to elaborate on the potential breakthroughs in pathogenesis. Important work continues regarding identification of an infectious etiology for KD. D ata were presented regarding the finding of cytoplasmic inclusion bodies in ciliated bronchial epithelium, suggestive of a previously unidentified RNA virus resulting in persistent infection. In addition, immunopathogenic mechanisms such as KD-associated genetic variant analysis, dysregulated immunological pathways and the role of innate and adaptive immunity, were discussed.
Although data remain inconclusive regarding treatment strategies for patients who do not respond to intravenous immune globulin (IVIG), postmarketing surveillance in Japan and recent reports all indicate that the coronary arteries of these patients are vulnerable and already damaged when the diagnosis of IVIG resistance is made. Attempts to identify these patients early and apply more aggressive primary therapy were advocated in the Yuki Lynn Takahashi memorial lecture. A state-ofthe art lecture on promises and pitfalls of clinical trials highlighted the need for, and challenges of, well-designed studies to address these unanswered questions. Experience with catheterbased coronary artery interventions guided by intravenous ultrasound was discussed in the Richard D Rowe memorial lecture. Two lectures addressed long-term aspects, including chronic anticoagulation for patients with giant aneurysms, and concerns and mechanisms regarding a potential acceleration of the atherosclerotic disease process late after the onset of $K D$. These informative and expert lectures have prompted all participants to expand their knowledge and increase teamwork to pave the way to solving the conundrum of $K D$.

\section{Epidemiology}

Epidemiologic features of $K D$ were reported from selected countries. In Japan, the 19th N ationwide Survey was conducted in 2007, and a total of 20,475 patients from 2005 and 2006 were reported. The incidence rate in 2006 was 188.1 per 100,000 children aged below 5 years, and it is estimated that if the rate continues to increase linearly, it will be over 200 in 2008. Seasonal peaks in the number of patients were observed in January and also in the summer season. In Taiwan, 
the annual incidence rate was 66 from 1996 to 2002, and 69 from 2003 to 2006 using $\mathrm{N}$ ational $\mathrm{H}$ ealth Insurance data. The number of patients increased in $M$ ay and June. $\mathrm{N} O$ increase of incidence rate was observed in the USA. Approximately 300 patients/year were reported to the $\mathrm{N}$ ational Passive Kawasaki Syndrome Surveillance from 1984 through 2004. Kids' Inpatient Database (KID) estimated the annual incidence rate at 19.6 in 2003. The rate in $\mathrm{H}$ awaii was 38.7 in 2005, but differed by race; 266.8 for Japanese, 94.8 for Chinese, 76.5 for $\mathrm{H}$ awaiians, 55.7 for Filipino, 11.4 for those of mixed race and 7.3 for Caucasians.

The annual incidence rates reported from selected countries were as follows; 8.39 in England (1998-2003), 24.3 in Ontario, Canada (2004-2006), 53 in $\mathrm{H}$ ong Kong (2000-2007), 36.8 in Shanghai (2002), 55.1 in Bejing, China (2004), 104.6 in Korea (2003-2005) with peaks between $M$ ay and July, approximately 5 in Thailand (1998-2002), 5.5 in France (1983-2007), 9.4 in Iceland (1998-2005), 10 in Finland (1998-2005), 7.4 in Sweden (1998-2005) and 5.2 in Norway (1998-2005). Interestingly, the incidence rate has increased in several countries, including Japan, England, Canada, Hong Kong and Korea, whereas in some other countries the rate did not increase, such as the USA and northern European countries. Patient reports from selected hospitals and other methods were reported from other countries. These include, 183 patients from Singapore without temporal and geographical clustering (1995-2007); several patients from India have been reported since 2005, 83 patients were diagnosed in a hospital in Jakarta, Indonesia (2000-2006), 63 patients from a hospital in Manila, Philippines, were reported (2006 and 2007) and six patients from Mongolia were reported through a nationwide survey.

\section{Genetics/genomics}

Progress in KD research was perhaps most dramatic in the area of genetics and gene expression, with the creation of large, multinational and multicenter collaborations finally allowing robust analysis of the genetic influences on KD susceptibility and disease outcome. O nouchi (Tokyo, Japan) presented the results of a Japan/USA collaboration that identified genetic variation in the inositol 1,4,5 trisphosphate kinase C (ITPKC) gene that affects both susceptibility to KD as well as risk of coronary artery aneurysms and IVIG resistance. The $C$ allele of the itpkc 3 single nucleotide polymorphism (SNP) leads to reduced splicing efficiency of the mRN A transcript and increased signaling through the calcineurin-nuclear factor of activated T cells (N FAT) pathway, resulting in increased levels of IL-2 production and T-cell activation. This work underscores the importance of $T$ cells in KD pathogenesis.

Two genome-wide association studies (GWAS) for KD susceptibility genes were reported from Taiwanese and northern European cohorts. Chen (Taipei, Taiwan) reported a GWAS in 254 Taiwanese KD subjects and 376 controls that identified 54 highly significant SN Ps $\left(p<10^{-10}\right)$. Burgner (Perth, Australia) reported the methods and preliminary analysis of a genome-wide association study by the International KD Genetics Consortium, which currently includes investigators and D N A samples from Singapore, The N etherlands, UK, Australia and USA. The analyses of these two GWAS are still pending. These presentations underscored the importance of a multicenter collaboration to amass sufficient numbers of samples required for both discovery and validation of implicated SN Ps.

Additional presentations by members of the International KD Genetics Consortium included the following presentations: Wright et al. (London, UK) reporting a novel method of pathway analysis of SN Ps in related genes that implicated genetic variation in the macrophage activation pathway in KD susceptibility; Breunis et al. (Amsterdam, The N etherlands) reporting a novel open reading frame in the $\mathrm{FC} \gamma$ receptor $2 \mathrm{C}$ whose expression was increased in $K D$ patients; Kuijpers et al. (Amsterdam,
The $\mathrm{N}$ etherlands) reporting on the role of VEG $F$ polymorphisms in KD susceptibility; and Burns et al. (CA, USA) reporting on the association of the itpkc_3 C allele with echocardiographic evidence of diastolic dysfunction.

G enetic associations that still need to be validated in independent cohorts include CTLA4 -318C/+49G/CT60G haplotypes (Chen et al., Taipei, Taiwan), FOXP3 rs3761548 A (Yang et al., Kaohsiung, Taiwan), CRP -286G/A (Liu et al., Shanghai, China), CCL11 -1328G/A (M atsubara et al., Yamaguchi, Japan), TNFRSF +36A/G (Fukazawa, Tokyo, Japan), FOXP3 543C/T (Olivito etal., Florence, Italy), the $\mathrm{H} L A$ region (Lee, Seoul, Korea), H LA-E rs2844724 (Lin et al., Taipei, Taiwan), KIR2D L2 (Oh et al., Seoul, Korea), MIF -173G/C (Simonini et al., Florence, Italy), IL-6 686C/G (Hong et al., Seoul, Korea), RANTES -403G/A (Chauduri, Chandigarh, India), PD-1 7210C/T (Chun et al., Seoul, Korea) and ACE I/D (Lijian, Shanghai, China).

Gene-expression studies reported by Abe et al. (Tokyo, Japan) found higher transcript abundance for haptoglobin, PRV-1, SORT-1, GAS7 and INTAM, and higher serum levels of granulocyte colony stimulating factor in KD patients who were resistant to IVIG therapy. Melish et al. (Honolulu, HI, USA) reported increased transcript abundance for genes involved in innate immunity, Toll-like receptor signaling, complement activation and cell adhesion. Notably absent were transcripts for type 1 interferon genes. The use of gene-expression patterns to identify candidate proteins for a diagnostic panel was also discussed.

\section{Pathogenesis/etiology}

Kajimoto et al. (U be, Japan) demonstrated that prostaglandin $\mathrm{E} 2$, which is elevated in acute KD sera, induces expression of $\beta 1$ integrin on cultured coronary artery endothelial cells via its receptor EP2, providing a potential therapeutic target for KD vasculitis. $H$ irono et al. (Toyama, Japan) examined cytokine levels in serum from children with refractory KD who received infliximab, demonstrating decreases in IL-6, 
transient increases in MRP8/MRP14 and TNF- $\alpha$, and no change in VEGF. $M$ ori et al. (Kanagawa, Japan) identified hyponatremia in $69 \%$ of 39 patients with acute KD and the syndrome of inappropriate antidiuretic hormone secretion in 31\% of these patients. They suggested that fluid restriction might be important in patients with the most severe hyponatremia. A study of disease associated genes and proteins in acute $K D$ revealed upregulation of genes associated with innate immune response, Toll-like receptor signaling, complement activation and matrix-adhesion molecules; upregulation of interferon signaling was not observed (Verma et al., Honolulu, USA). Suzuki et al. (Wakayama, Japan) demonstrated greater T-cell activation as measured by serum levels of soluble IL-2 receptor and IL-6 in IVIG nonresponders. A combination of high serum TNF- $\alpha$ and low VEGF-D levels was found to be characteristic of KD patients who developed coronary artery disease. Regulation of lymphangiogenesis through VEGF-D was proposed as a possible therapeutic strategy in acute KD (Ebata, Japan). There were several presentations on the Lactobacillus casei-induced murine model of coronary arteritis. These included presentations by $A n$ et al. (Toronto, Canada), on IL-6-mediated elastin breakdown; Schulte et al. (LoS Angeles, CA, USA), on the requirement for $T$ cells, macrophages and dendritic cells but not B cells in the process; Lin et al. (Kaohsiung, Taiwan), on Toll-like receptor-mediated arteritis and Salazar et al. (H ouston, TX, USA), on differences in size-fractionated preparations on the induction of arteritis versus cholangitis. Serum-induced rabbit (O zawa et al., Kyoto, Japan) and swine models (Philip et al., Chennai, India) were also discussed. Whitin et al. (Palo Alto, CA, USA) described the elevation of a novel truncated form of serum amyIoid $A$ as a potential biomarker to distinguish KD from other pediatric febrile illnesses. Furthermore, many interesting posters were presented, including a description of new molecular methods to determine a possible viral etiology of
KD (Shike et al., San Diego, CA, USA) and the use of human airway epithelial cell culture to attempt to cultivate an etiologic agent of KD (Baker et al., Chicago, IL, USA).

\section{Pathology, animal models}

\section{$\&$ diagnosis}

It was impressive that many analyses using animal vasculitis models were reported at this symposium. Philip et al. (Chennai, India) injected horse serum to swine to induce immune complextype vasculitis. O zawa et al. (Kyoto, Japan) also reported establishing a rabbit vasculitis model through the injection of horse serum. Analyses of a murine vasculitis model induced with a L. casei cell wall extract were reported by many investigators (An et al., Schulte et al., Lin et al. and Salazar et al.). O haraseki et al. (Tokyo, Japan) and Takahashi et al. (Tokyo, Japan) presented the histological findings from a mouse vasculitis model induced with Candida albicans water-soluble fractions. The advantages and disadvantages of each experimental model as animal models of KD were discussed.

U ehara et al. (Tochigi, Japan) reported the epidemiologic characteristics of KD patients with changes in the skin at the bacille Calmette-Guerin (BCG) inoculation site. They demonstrated that more than $70 \%$ of patients aged 3-20 months had redness at the BCG inoculation site. They concluded that redness at the $B C G$ site may be a useful observation supporting the diagnosis of KD. Yellen et al. (Boston, M A, USA) retrospectively reviewed the results of application of the American $\mathrm{H}$ eart Association 2004 recommendations to KD patients with coronary artery aneurysms. They stressed that the recommendations improved recognition and treatment of KD by applying the algorithm to patients suspected of having incomplete KD.

\section{Acute management}

$M$ any studies presented at the symposium confirmed earlier observations that approximately $15 \%$ (range: $10-20 \%$ ) of KD patients have persistent or recrudescent fever after their first IVIG infusion ('IVIG resistance'). This group is at significantly higher risk for development of coronary aneurysms. M atsubara at al. reported that the IgG allotype GIM (2) tended to be associated with IVIG resistance. Abstracts on treatment in the acute phase focused on either primary treatment with IVIG and adjunctive anti-inflammatory agents, or on secondary treatment of IVIG-resistant patients. With respect to primary treatment, Suzuki et al. (Tokyo, Japan) suggested that primary treatment with IVIG and a tapering course of intravenous and then oral corticosteroids was more effective than IVIG alone in patients who were stratified into a high-risk group at baseline using the Kobayashi score. In a multicenter study in Japan, O gihara et al. validated the Kurume score for IVIG resistance, reporting a $73 \%$ sensitivity and $92 \%$ specificity. $\mathrm{H}$ owever, Tremoulet etal. (San Diego, CA, USA) reported that risk stratification for IVIG resistance using the Egami score developed in Japan did not work well in a genetically diverse US population. Among presentations on treatment of IVIG-resistant KD, Latino et al. (Toronto, Canada) found that coronary artery and fever outcomes worsened among patients whose first retreatment was with steroids rather than IVIG, and the inverse among patients requiring a third treatment. Perhaps most importantly, patients requiring multiple retreatments had worsened coronary outcomes. Suzuki et al. (Tochigi, Japan) reported the results of various retreatment regimens using additional IVIG and steroids from the first and second investigations of the 19th $\mathrm{N}$ ationwide Survey of KD in Japan. The incidence of coronary lesions was greater than 30\% among IVIG-resistant patients whose first retreatment was with IVIG and second retreatment was with steroids. Patients in whom both a first and second retreatment was with steroids also demonstrated a high rate of coronary lesions $(24 \%)$. The authors concluded that additional steroids might be related to worsening coronary status, but could not exclude residual confounding. 
The symposium featured many presentations on the role of TN F- $\alpha$ in the pathogenesis of KD; the role of T N F- $\alpha$ antagonists in treatment of KD was of particular interest. Burns et al. (San Diego, CA, USA) reported a small $(n=24)$ prospective, multicenter, randomized safety and pharmacokinetic study of a TNF- $\alpha$ antagonist (infliximab) in the treatment of refractory KD. Apart from unexplained hepatomegaly, there were no significant adverse effects of infliximab treatment. In a small multicenter retrospective series of patients in Korea, Song et al. reported that infliximab treatment in IVIG-resistant patients approved successful in abating fever and possibly in arresting further deterioration of coronary lesions. Finally, Portman et al. (Seattle, WA, USA) reported a prospective, open-label study of the safety and pharmacokinetics of etanercept for the primary treatment of KD. Etanercept appeared to be safe, with pharmacokinetics similar to those observed in older patients with juvenile rheumatoid arthritis. Larger, prospective, randomized studies will be needed for definitive assessment of the safety and efficacy of treatment with T N F- $\alpha$ antagonists.

\section{Management of complications}

Kanamaru et al. (Tokyo, Japan) reported that multislice spiral computed tomography can reliably evaluate coronary artery lesions, including assessment of intimal proliferation and calcification within aneurysms. Katsumata et al. (Tokyo, Japan) used magnetic resonance coronary angiography to visualize and classify types of coronary artery segmental stenoses. Magnetic resonance myocardial imaging was also useful in evaluating myocardial ischemia and the viability of the myocardium supplied by branches with segmental stenoses. The prevalence of coronary artery fistula in the acute and subacute phases of KD was estimated to be $5.3 \%$ by echocardiography (Liang et al., Kaohsiung, Taiwan). Takahashi (Los Angeles, CA, USA) reported that use of warfarin and aspirin for management of giant coronary aneurysms results in improved event-free survival compared with treatment with aspirin alone. The combination of antithrombin and antiplatelet therapies was more effective than antiplatelet therapy alone. $M$ anlhiot et al. (Toronto, Canada) reported 36 patients with severe coronary artery aneurysms treated with systemic anticoagulation. Use of low molecular weight heparin was associated with a greater degree of vascular remodeling compared with warfarin. They suggested that low molecular weight heparin should be used initially and later switched to warfarin in order to maximize the potential for coronary artery aneurysm regression. Suda et al. (Kurume, Japan) noted that the timerelated freedom from cardiac events for 70 patients with giant coronary artery aneurysms at 1, 5, 10 and 20 years was $71,59,54$ and 29\%, respectively. Various types of catheter and surgical interventions were required in up to half of these patients. Sabharwal et al. (Toronto, Canada) demonstrated that greater rates of regression were related to aneurysm characteristics (maximal size, location and shape), younger age at KD and male gender. A further study from this group showed a freedom from progression to stenoses of only $57 \%$ after 5 years. Ishii (Sagamihara, Japan) reported from a multicenter study in Japan that percutaneous coronary artery interventions for obstructions can be accomplished and are effective in the long term, and may be equivalent to coronary artery bypass graft surgery.

\section{Long-term outcomes}

After the acute episode, parents and patients express considerable anxiety regarding long-term prognosis, as noted by $C$ hahal et al. (Toronto, Canada). For all KD patients, particularly those with important ongoing coronary artery involvement, the risk of accelerated atherosclerosis continues to be of concern. Mechanisms of latestage arteritis and atherosclerosis are now being explored in animal models of $K D$. It was suggested that there was a need to differentiate between arteriosclerosis (occurring at sites of coronary artery lesions) and atherosclerosis (occurring at sites of transition to normal segments). Lin et al. (Taipei, Taiwan) demonstrated evidence of elevated serum markers indicative of abnormal matrix remodeling in patients with and without persistent lesions. M itani et al. (Tsu, Japan) used virtual histology intravascular ultrasound to assess coronary artery lesions and noted features potentially consistent with advanced atherosclerotic placques, particularly in areas of stenoses. N oninvasive vascular markers continue to be a tool for investigation. Liu et al. demonstrated increased carotid intima-media thickness in KD patients with persistent lesions, with significantly reduced numbers of circulating endothelial progenitor cells, a marker of endothelial repair and neovascularization. However, controversy continues as to whether abnormalities are present in those KD patients without lesions. $\mathrm{N}$ iboshi et al. (Kyoto, Japan) noted impaired brachial artery reactivity (endothelial function) and increased markers of oxidative stress in KD patients regardless of the degree of coronary artery involvement. By contrast, Ishii et al. (Kurume, Japan) noted abnormal carotid artery stiffness and distensibility only in KD patients with lesions. Novel risk factors for atherosclerosis were observed in KD patients regardless of the presence coronary artery lesions (Fukunaga et al., Juntendo, Japan). Screening, management and ongoing counseling regarding cardiovascular risk is recommended for all patients. Lin et al. (Toronto, C anada) demonstrated very low levels of physical activity in KD patients that produce an impact on functional health status and may be influenced by uncertainties about coronary artery disease and anticoagulation. Statin therapy may play a role owing to its pleotropic effects on inflammation. Shih-M ing et al. (Kaohsiung, Taiwan) treated 11 children with lesions with 
statin and noted improved inflammatory markers and endothelial function. $\mathrm{H}$ amaoka et al. (K yoto, Japan) treated eight patients with lesions and noted similar improvements.

These small, early studies suggest that further investigation is required on the potential role of statins for long-term management of KD.
Financial \& competing interests disclosure

Theauthorshaveno revant affiliationsor financial involvement with any organization or entity with a financial interest in or financial conflict with the subject matter or materials discussed in the manuscript. Thisindudesemployment, consultancies, honoraria, stock ownership or options, expert testimony, grants or patents received or pending, or royalties
No writing assistance was utilized in the production of this manuscript.

\section{Website}

101. Taiwan Society of Pediatric Cardiology www.tspc.org.tw/IX_IKDS_Abstracts/ abstracts.pdf 